\title{
Persistent Primitive Olfactory Artery in Serbian Population
}

\author{
Ljiljana Vasović, ${ }^{1}$ Milena Trandafilović, ${ }^{2}$ Slobodan Vlajković, ${ }^{1}$ \\ Ivan Jovanović, ${ }^{1}$ and Slađana Ugrenović ${ }^{1}$ \\ ${ }^{1}$ Department of Anatomy, Faculty of Medicine, University of Niš, Boulevard Dr. Zoran Đinđić 81, 18000 Niš, Serbia \\ ${ }^{2}$ Center for Biomedical Researches, Faculty of Medicine, University of Niš, Boulevard Dr. Zoran Đinđić 81, 18000 Niš, Serbia \\ Correspondence should be addressed to Ljiljana Vasović; likica@medfak.ni.ac.rs
}

Received 11 April 2013; Accepted 17 June 2013

Academic Editor: Hongjuan Liu

Copyright (C) 2013 Ljiljana Vasović et al. This is an open access article distributed under the Creative Commons Attribution License, which permits unrestricted use, distribution, and reproduction in any medium, provided the original work is properly cited.

\begin{abstract}
The continuation of the cranial branch of the primitive internal carotid artery is called the primitive olfactory artery (PO $\ell \mathrm{A})$. It takes this name according to the fact that it is mainly concerned with supplying the developing nasal region. We reported two new cases of the persistent PO $\ell$ A (PPO $\ell$ A) in Serbian population after retrospective analysis of digital images of 200 fetal and 269 adult cases. This PPO $\ell$ A originated from the precommunicating part (A1) of the right anterior cerebral artery, coursed along the olfactory tract, and turned on the medial cerebral hemisphere in both male adults. Some vascular variations (fenestration of the Al and the median artery of the corpus callosum) were associated with this persistent vessel. According to the fact that we did not find aneurysm in our previous and two recent cases, we are of the opinion that PPO $\ell$ A is usually asymptomatic in Serbian population.
\end{abstract}

\section{Introduction}

During embryonic development of the vascular system, the primitive olfactory artery ( $\mathrm{PO} \ell \mathrm{A})$ represents a continuation of the cranial branch of the primitive internal carotid artery (ICA) [1]. It may be presumed that a fenestration of the anterior cerebral artery (ACA) is the remnant of the embryonal plexiform anastomosis between the ACA and the PO $\ell$ A $[2,3]$. Some authors noted that $\mathrm{PO} \ell \mathrm{A}$ normally regresses to the remnant-the recurrent artery of Heubner (RAH) $[4,5]$. Other authors supported this claim by the evidence of an aplasia of the RAH [6-8] and/or the anterior communicating artery $[6,9]$.

A persistence of the $\mathrm{PO} \ell \mathrm{A}(\mathrm{PPO} \ell \mathrm{A})$ is very rare according to the fact that its incidence was noted in $0.14 \%$ [10] to $0.29 \%$ [11] of cases.

Morphologically, the PPO $\ell$ A usually courses anteromedially along the ipsilateral olfactory tract, and after a turn, it supplies the ACA territory [4]. Pathoanatomically, a relatively frequent location of an aneurysm on the hairpin bend of the PPO $\ell$ A emphasizes the importance of hemodynamic stress in this persistent primitive vessel $[7,12,13]$.

Reports of single cases or retrospective studies about special and/or general features of the PPO $\ell$ A came usually from Japan [5-10, 12-17] and Korea [11, 18, 19], although there were case reports from UK [20], Taiwan [21], and Serbia [22].

Previous incidental finding of the PPO $\ell \mathrm{A}$ in one adult cadaver [22], and the description of fenestration of the precommunicating part (A1) of the ACA [3] and/or RAH in fetuses [23], inspired us to a more detailed investigation of the $\mathrm{PO} \ell \mathrm{A}$ persistence in Serbian population.

\section{Materials and Methods}

We did a retrospective analysis of digital images of brain vessels of 200 fetal and 269 adult cadavers, dissected at the Department of Anatomy and Institute of Forensic Medicine in Niš, respectively.

2.1. Fetal Cadavers. Fetuses of both genders, from 9 to 32 weeks of gestation, were a part of the collection of our Department of Anatomy, and they were used in the preparation of doctoral thesis [24]. All fetuses were obtained legally from the Clinic of Gynecology and Obstetrics in Nišs. The Council for Postgraduate Study of the Faculty of Medicine in Niš gave permission to investigate the fetal material. The arteries of fetuses were injected with Micropaque or latex 
through the left cardiac ventricle or through the common carotid artery. All fetuses were fixed in 10\% formalin for 2 weeks. Fetal brains were removed and kept in individual calvarias. The measurements were performed by means of an ocular micrometer mounted on an operating microscope (Olympus).

2.2. Adult Cadavers. The dissected brains originated from cadavers of both genders, different ages (from the neonate to 95 years), and different causes of death in the period between 2006 and 2013. Investigation of these cases was in accordance with the rules of the internal Ethics Committee (no. 01-90684) of our Faculty of Medicine. Morphological features of brain arteries (caliber, possible vessel's abnormalities) were observed by using a magnifying glass and recorded on a film. Measurement of the external diameter of arteries was performed by ImageJ (http://rsb.info.nih.gov/ij/index.html).

\section{Results and Discussion}

3.1. Results. We discovered two new cases of the PPO $\ell \mathrm{A}$ in adults. The first case of the PPO $\ell A$ was found in a male cadaver, aged 58 and autopsied due to cardiac arrest; another case was found in a male, aged 61, who died due to polytrauma at the Orthopedic Clinic.

(1) Case I. The PPO $\ell$ A had a common origin with the $\mathrm{RAH}$ from the A1 of the right ACA at the level of the proximal part of the fenestration (Figure 1(a)). Its beginning was about $7 \mathrm{~mm}$ away from ICA bifurcation. The caliber of the right A1 was $2.03 \mathrm{~mm}$, whereas the caliber of the PPO $\ell A$ was $1.41 \mathrm{~mm}$. The latter followed the olfactory tract in the first part of its course, and after that, it turned and passed on the medial telencephalic surface. However, we did not photograph its termination. In addition, the median artery of the corpus callosum was also presented (Figure 1(b)).

(2) Case II. The PPO $\ell \mathrm{A}$ was a branch of the right A1 (Figure 2(a)). The beginning of the PPO $\ell \mathrm{A}$ was about $6 \mathrm{~mm}$ away from ICA bifurcation. The caliber of the right Al was $2.50 \mathrm{~mm}$, whereas caliber of the $\mathrm{PPO} \ell \mathrm{A}$ was $1.40 \mathrm{~mm}$. The latter followed the olfactory tract in the first part of its course, and after that, it passed similar to the callosomarginal artery; a bihemispheric branch was also evidenced at the level of its termination (Figure 2(b)). Ipsilaterally, the RAH originated from the PPO $\ell$ A it was duplicated at the beginning. Atherosclerotic plaques were significantly present at the cerebral arteries, especially along main brain arteries.

(3) Comparison of PPO $\mathrm{AAs}$ in the Literature. General and special data about PPO $\ell$ As in our and other populations are presented in Table 1 .

3.2. Discussion. Firstly, we noted some data and disagreements in the literature about the PO $\ell$ A origin $[1,6,25], \mathrm{PO} \ell \mathrm{A}$ rudiments [1] or a lack of embryologic explanation of A1 variable side branches $[15,26]$, as well as an origin [20] or termination $[8,15]$ of the PPO $\ell$ A.

Moffat [1] described that PO $\ell$ A has a similar development in the rat's and human embryos up to an $18 \mathrm{~mm}$ stage. During the development of vascular system in a $3.7 \mathrm{~mm}$ embryo, the continuation of the dorsal aorta forms the primitive ICA [1], except for its first segment which was formed by the primitive third aortic arch [6]. At the level of the forebrain the ICA gives the primitive maxillary artery, and then ICA divides into the cranial and caudal branches. In embryos of 4 to $5.7 \mathrm{~mm}$ (28-30 days), the cranial branch constitutes the primitive olfactory artery (PO $\ell \mathrm{A})$, which branches off the anterior choroidal and middle cerebral arteries [6]. According to data from the paper by Horie et al. [13], the definitive ACA extends superiorly between the cerebral hemispheres, associated with regression of the PO $\ell \mathrm{A}$ untill the 7 th week of gestation. According to the picture of human embryo in the paper by Katayama et al. [14], the PO $\ell$ A retains its origin from the A1 to the $9 \mathrm{~mm}$ embryonic stage. Komiyama [25] and Okahara et al. [6] stressed that the PO $\ell$ A terminates in the nasal fossa, and "secondary artery" constitutes the medial olfactory artery, which supplies the olfactory bulb. This medial olfactory artery becomes the ACA proper in an 11.5 to $18 \mathrm{~mm}$ embryo, while the terminal portion of the PO $\ell A$ usually regresses. Kim et al. [27] noted the existence of the plexiform anastomosis between the ACA and the PO $\ell A$ in the illustration of a $14 \mathrm{~mm}$ embryo. Lateral olfactory branches of the $\mathrm{PO} \ell \mathrm{A}$ include the $\mathrm{RAH}$, anterior choroidal, lateral striate, and middle cerebral arteries. Moffat [1] noted that the PO $\ell A$ in a $24 \mathrm{~mm}$ human embryo forms an inconstant striate branch of the ACA.

Okahara et al. [6] noted that the PPO $\ell A$ arises only from the A1 part, as in cases described by Moffat [20] and Horie et al. [13], as well as in recent cases. In addition, we could compare the origin of PPO $\ell \mathrm{A}$ from the $\mathrm{A} 1$ part in these cases with the PO $\ell A$ origin in an $18 \mathrm{~mm}$ human embryo whose picture was displayed in the paper by Katayama et al. [14]. Tsutsumi et al. [8] described that PPO $\ell$ A originated from A1-A2 junction. However, many authors described its internal carotid origin $[7,9,14,16,19,21]$. In our previous case the PPO $\mathrm{A}$ and posterior communicating artery had a common origin from the middle cerebral artery (MCA) [22]. It can be said that the case, described by Lin et al. [21], in which PPO $\ell$ A continued from an accessory MCA, resembles our case. Interesting anomaly was found in five rat embryos studied. In these specimens the cranial branch of the ICA terminated as the MCA, whereas the PO $\ell$ A was a continuation of the primitive maxillary artery [1].

Several types of the PPO $\ell \mathrm{A}$ are described in the literature. In the first type described by Nozaki et al. [7] the PPO $\ell A$ rose from the ICA, ran along the olfactory tract, and made a hairpin bend to supply the territory of the ACA postcommunicating part (A2). In the second type, described by the same authors, the PPO $\ell$ A rose from the ACA and passed through the cribriform plate of the ethmoid bone to supply the nasal cavity, similar to the ethmoidal arteries. The case described by Enomoto et al. [15], on computer tomography angiography (CTA), and the case described by Moffat [20] on autopsy were classified as the second type. Komiyama [25] noted that 


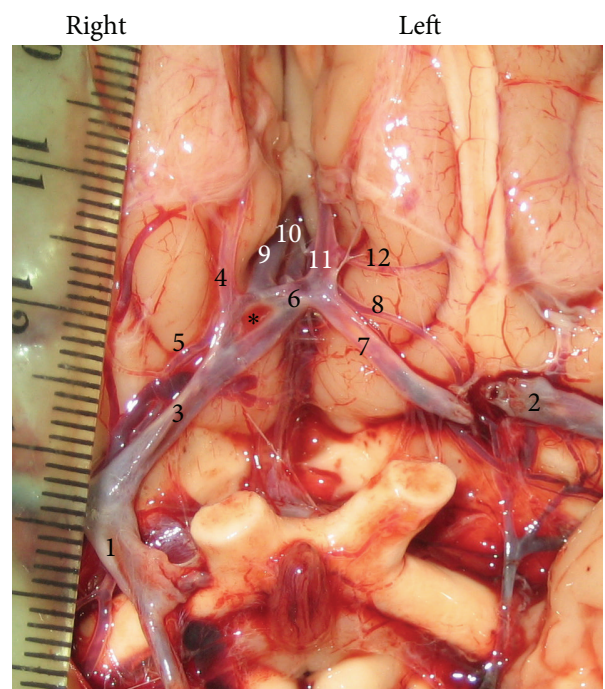

(a)

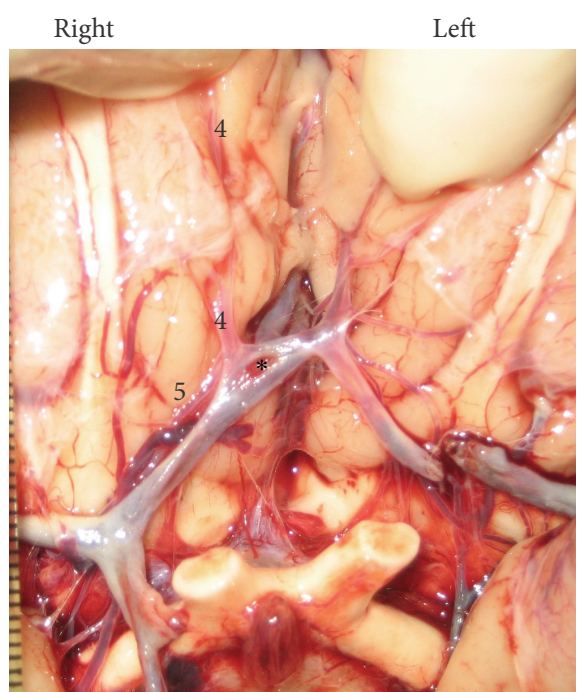

(b)

FIGURE 1: Persistent primitive olfactory artery (PPO $\ell$ A) as a side branch of the precommunicating part (A1) of the right anterior cerebral artery (ACA). It originates at the level of proximal end of the A1 fenestration (a) and courses to the medial surface of the frontal lobe (b). Cerebral part (C4) of the right internal carotid artery (1); left C4 (2); right A1 (3); A1 fenestration (*); right PPO $\ell$ A (4); right recurrent artery of Heubner (RAH) (5)); anterior communicating artery (ACoA) (6)); left A1 (7); left RAH (8); right postcommunicating part (A2) of the ACA (9); median artery of the corpus callosum (10); left A2 (11); left medial frontoorbital artery (12).

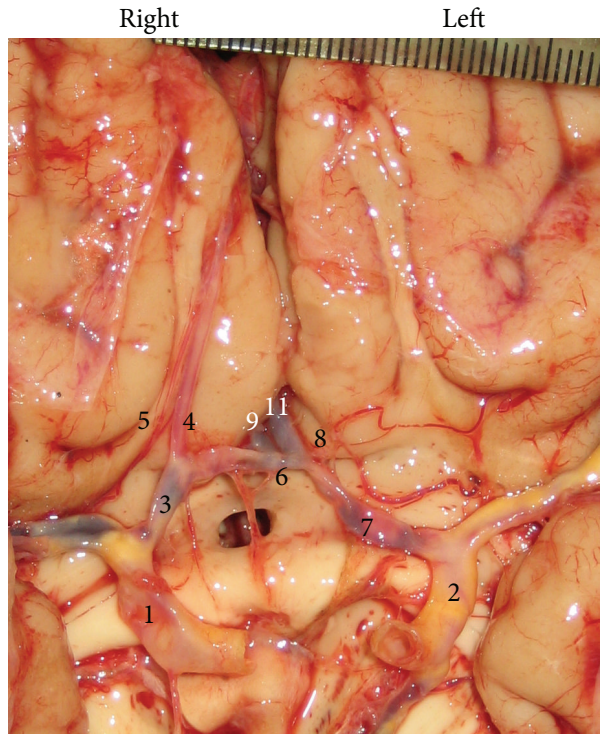

(a)

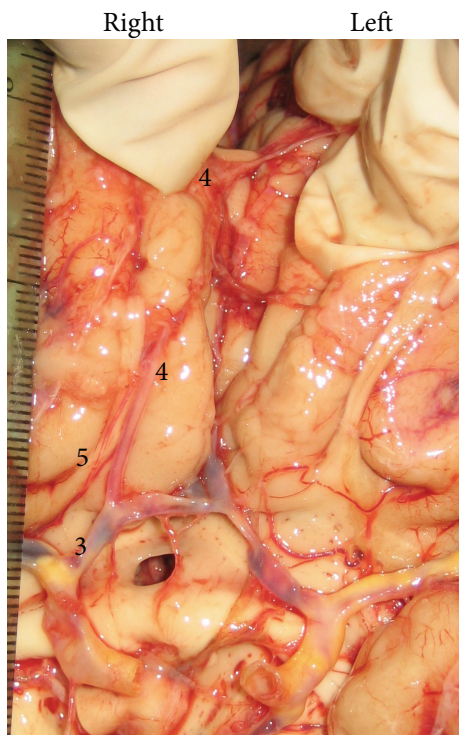

(b)

FIGURE 2: Persistent primitive olfactory artery (PPO $\ell A$ ) as a side branch of the precommunicating part (A1) of the right anterior cerebral artery (ACA). It originates from the anterior wall of the A1 (a) and courses to the medial surface of the frontal lobe, where the PPO $\ell \mathrm{A}$ gave off a bihemispheric branch (b). Cerebral part (C4) of the right internal carotid artery (1); left C4 (2); right A1 (3); right PPO $\ell$ A (4); right recurrent artery of Heubner (RAH) (5)); anterior communicating artery (ACoA) (6); left A1 (7); left RAH (8); right postcommunicating part (A2) of the ACA (9); left A2 (11).

this second type of the $\mathrm{PPO} \ell \mathrm{A}$ is homologous to the internal ethmoidal artery in the dog. In the third (transitory) type described by Horie et al. [13], the PPO $\ell$ A divided into two branches; one artery was similar to Nozaki's type 1, whereas the second one had features of Nozaki's type 2. In our "third" type, the $\mathrm{PPO} \ell \mathrm{A}$ of the MCA origin had a common trunk with posterior communicating artery ( $\mathrm{PCoA})$ and coursed forward to the ipsilateral olfactory tract [22]. The case of the $\mathrm{PPO} \ell \mathrm{A}$ termination (PPO $\ell \mathrm{A}$ - cortical frontal vein shunt) described by Tsutsumi et al. [8] was classified as type 4 .

Medial frontoorbital and frontopolar arteries are different from the PPO $\ell \mathrm{A}$ according to the beginning (A2 part) and 


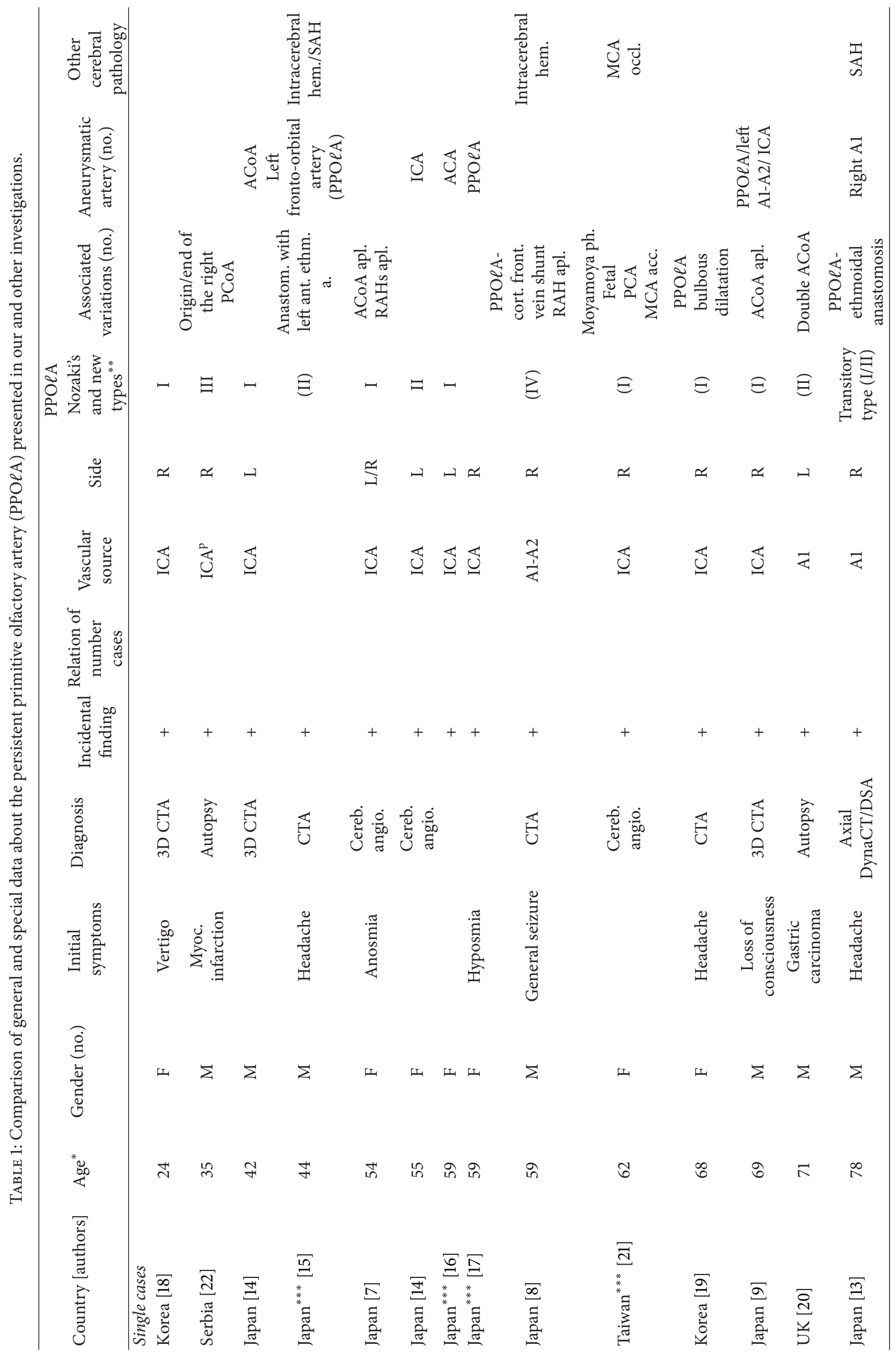




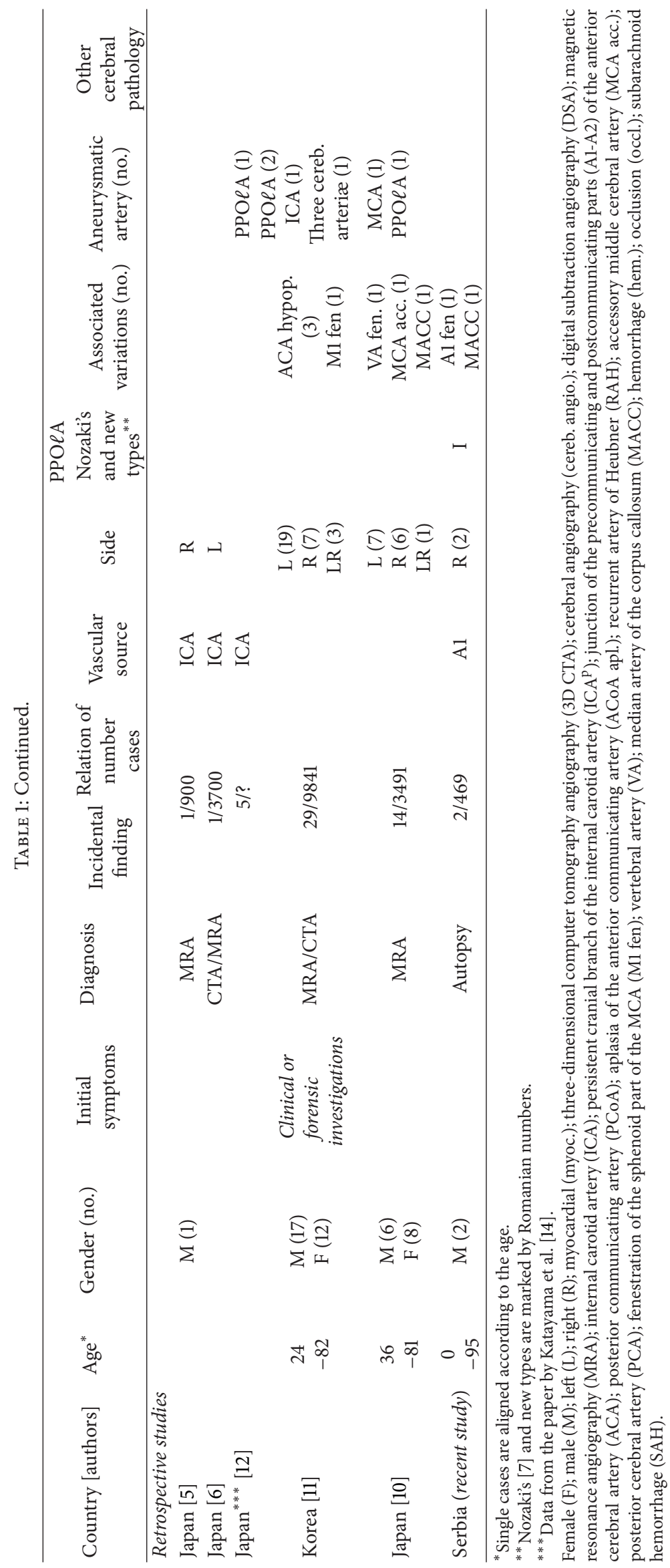


their course. Exceptions were possible as in cases described by Enomoto et al. [15] and Krishnamoorthy et al. [26].

We described some cases of the PO $\ell$ A partial persistence in human fetuses [23]. Recently, we described these two cases of the PPO $\ell$ A, found only among adult cadavers. Previous [22] and recent cases indicate that the incidence of the PPO $\ell A$ is $0.64 \%$ in Serbian population. These incidences were $0.14 \%$ in the Japanese population [10] and $0.29 \%$ in the Korean population [11]. For the purpose of calculating of number of published cases, we included some paper abstracts [12, 14-17] in the list of references. Based on the case numbers in our Table 1 and Moffat's allegation about two cases of the PPO $\ell A$ described in 1951 and 1961 by other authors [1], we counted 67 cases to this time. Based also on the cases in the same table, we could note that $\mathrm{PPO} \ell \mathrm{A}$ was more frequent in males and on the left side, although the PO $\ell$ A persisted on the right side in males of Serbian population, as we noted in our work. The existence of bilateral PPO $\ell A$ was noted five times in the literature $[7,10,11]$. The youngest person was 18 years old [5] and the oldest 82 years [11].

In summary, we did not find any aneurysm on the PPO $\ell A$ in our cases, opposite to some authors who found it on the PPO $\ell A$ hairpin bend $[9,12,15,17]$, and/or on other cerebral arteries $[9-11,13,14,16]$. Association of the PPO $\ell A$ and other vascular variations were also noted $[7,11,20,22]$, as in our adult cases.

\section{Conclusions}

The primitive olfactory artery is a rare persistent primitive vessel $(0.64 \%)$ in Serbian population. It was incidental finding in presented cases. We did not find any complete vascular trunk in fetuses, but we did in adults of male gender, on the right side.

\section{Acknowledgments}

The authors wish to thank Mrs Zorica Antić, Professor of English in Faculty of Medicine of Niš, for assistance in editing the text. Contract Grant sponsor is Ministry of Education, Science and Technological Development of Republic of Serbia (nos. 41018 and 175092).

\section{References}

[1] D. B. Moffat, "The embryology of the arteries of the brain. Arris and Gale lecture delivered at the Royal College of surgeons of England," pp. 368-382, 1962, http://www.ncbi.nlm.nih .gov/pmc/articles/PMC2414182/pdf/annrcse00385-0020.pdf.

[2] T. Minakawa, M. Kawamata, M. Hayano, and K. Kawakami, "Aneurysms associated with fenestrated anterior cerebral arteries. Report of four cases and review of the literature," Surgical Neurology, vol. 24, no. 3, pp. 284-288, 1985.

[3] J. S. Koh, S. H. Lee, J. S. Bang, and G. K. Kim, "Threedimensional angiographic demonstration of plexiform fenestrations of the proximal anterior cerebral artery associated with a ruptured aneurysm," Journal of Korean Neurosurgical Society, vol. 44, no. 5, pp. 338-340, 2008.
[4] S. J. Dimmick and K. C. Faulder, "Normal variants of the cerebral circulation at multidetector CT angiography," Radiographics, vol. 29, no. 4, pp. 1027-1043, 2009.

[5] A. Uchino, A. Sawada, Y. Takase, and S. Kudo, "MR angiography of anomalous branches of the internal carotid artery," American Journal of Roentgenology, vol. 181, no. 5, pp. 1409-1414, 2003.

[6] M. Okahara, H. Kiyosue, H. Mori, S. Tanoue, M. Sainou, and $\mathrm{H}$. Nagatomi, "Anatomic variations of the cerebral arteries and their embryology: a pictorial review," European Radiology, vol. 12, no. 10, pp. 2548-2561, 2002.

[7] K. Nozaki, W. Taki, O. Kawakami, and N. Hashimoto, "Cerebral aneurysm associated with persistent primitive olfactory artery aneurysm," Acta Neurochirurgica, vol. 140, no. 4, pp. 397-402, 1998.

[8] S. Tsutsumi, Y. Shimizu, Y. Nonaka et al., "Arteriovenous fistula arising from the persistent primitive olfactory artery with dual supply from the bilateral anterior ethmoidal arteries-case report," Neurologia Medico-Chirurgica, vol. 49, no. 9, pp. 407409, 2009.

[9] T. Yamamoto, K. Suzuki, T. Yamazaki, W. Tsuruta, T. Tsurubuchi, and A. Matsumura, "Persistent primitive olfactory artery aneurysm-case report," Neurologia Medico-Chirurgica, vol. 49, no. 7, pp. 303-305, 2009.

[10] A. Uchino, N. Saito, E. Kozawa, W. Mizukoshi, and K. Inoue, "Persistent primitive olfactory artery: MR angiographic diagnosis," Surgical and Radiologic Anatomy, vol. 33, no. 3, pp. 197-201, 2011.

[11] B. R. Kwon, S. H. Yeo, H. W. Chang et al., "Magnetic resonance angiography and CT angiography of persistent primitive olfactory artery: incidence and association rate with aneurysm in Korea," Journal of the Korean Society of Radiology, vol. 66, no. 6, pp. 493-499, 2012.

[12] T. Kaneko, K. Suetake, T. Shinya, and M. Takeda, "Persistent primitive olfactory artery: report of 5 cases," Neurological Surgery, vol. 27, no. 10, pp. 933-939, 1999.

[13] N. Horie, M. Morikawa, S. Fukuda, K. Hayashi, K. Suyama, and I. Nagata, "New variant of persistent primitive olfactory artery associated with a ruptured aneurysm," Journal of Neurosurgery, vol. 117, no. 1, pp. 26-28, 2012.

[14] M. Katayama, Y. Shimamoto, and S. Suga, "Primitive olfactory artery-characteristics and cerebral aneurysms associated with primitive olfactory artery," Niche Neuro-Angiology Conference, (Jpn/Eng), 2012, http://nnac.umin.jp/nnac/ Download_2012_files/Katayama.pdf.

[15] H. Enomoto, H. Goto, and M. Murase, "A ruptured cerebral aneurysm of the frontoorbital artery and the coexisting anastomosis with the anterior ethmoidal artery. A case report," Neurological Surgery, vol. 14, no. 2, pp. 203-206, 1986.

[16] T. Tsuji, M. Abe, and K. Tabuchi, "Aneurysm of a persistent primitive olfactory artery: case report," Journal of Neurosurgery, vol. 83, no. 1, pp. 138-140, 1995.

[17] A. Yamaura, M. Takase, and H. Makino, "An aneurysm of the proximal anterior cerebral artery at the olfactory bulb," Surgical Neurology, vol. 12, no. 5, pp. 425-427, 1979.

[18] M. S. Kim and G. J. Lee, "Diagnosis of persistent primitive olfactory artery using computed tomography angiography," Journal of Korean Neurosurgical Society, vol. 49, no. 5, pp. 290291, 2011.

[19] S. H. Yeo, B. H. Rho, E. Kim, C. H. Sohn, and H. W. Chang, "Diagnosis of persistent primitive olfactory artery using CT angiography: a case report," Journal of the Korean Society of Radiology, vol. 61, no. 3, pp. 139-141, 2009. 
[20] D. B. Moffat, "A case of peristence of the primitive olfactory artery," Anatomischer Anzeiger, vol. 121, no. 5, pp. 477-479, 1967.

[21] W.-C. Lin, S.-W. Hsu, Y.-L. Kuo, J. A. Feekes, and H.-C. Wang, "Combination of olfactory course anterior cerebral artery and accessory middle cerebral artery (MCA) with occluded in situ MCA and related moyamoya phenomenon," Brain and Development, vol. 31, no. 4, pp. 318-321, 2009.

[22] L. Vasović, I. Jovanović, S. Ugrenović, and M. Trandafilović, "Remnants of embryonic arteries on the brain base: a case report," World Journal of Neuroscience, vol. 2, no. 2, pp. 133-137, 2012.

[23] L. Vasović, S. Ugrenović, and I. Jovanović, "Human fetal medial striate artery or artery of Heubner," Journal of Neurosurgery Pediatrics, vol. 3, no. 4, pp. 296-301, 2009.

[24] L. Vasović, Morphological characteristics of the cerebral arterial circle with different origin of the vertebral arteries [M.S. thesis], Faculty of Medicine, Nišs, Serbia, 1990.

[25] M. Komiyama, "Persistent primitive olfactory artery," Surgical and Radiologic Anatomy, vol. 34, no. 1, pp. 97-98, 2012.

[26] T. Krishnamoorthy, A. K. Gupta, R. N. Bhattacharya, B. J. Rajesh, and S. Purkayastha, "Anomalous origin of the callosomarginal artery from the Al segment with an associated saccular aneurysm," American Journal of Neuroradiology, vol. 27, no. 10, pp. 2075-2077, 2006.

[27] T. H. Kim, H. K. Lee, J. J. Rhee, S. J. Lee, C. H. Lee, and M. S. Kim, "The incidence and clinical significance of fenestrations in the horizontal segment of the anterior cerebral artery detected by conventional angiography and magnetic resonance angiography," Journal of Korean Neurosurgery Society, vol. 40, no. 1, pp. 74-78, 2006. 

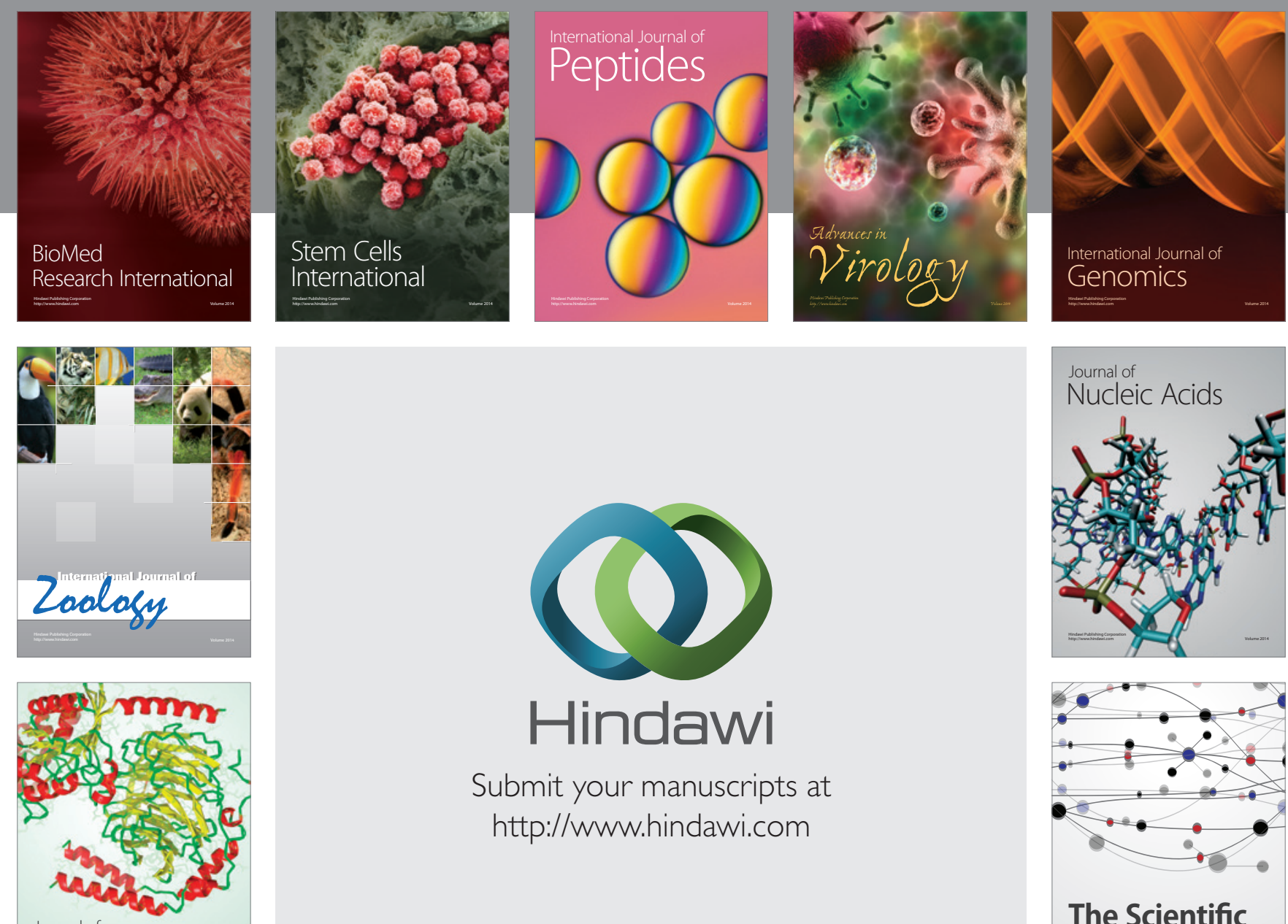

Submit your manuscripts at

http://www.hindawi.com

Journal of
Signal Transduction
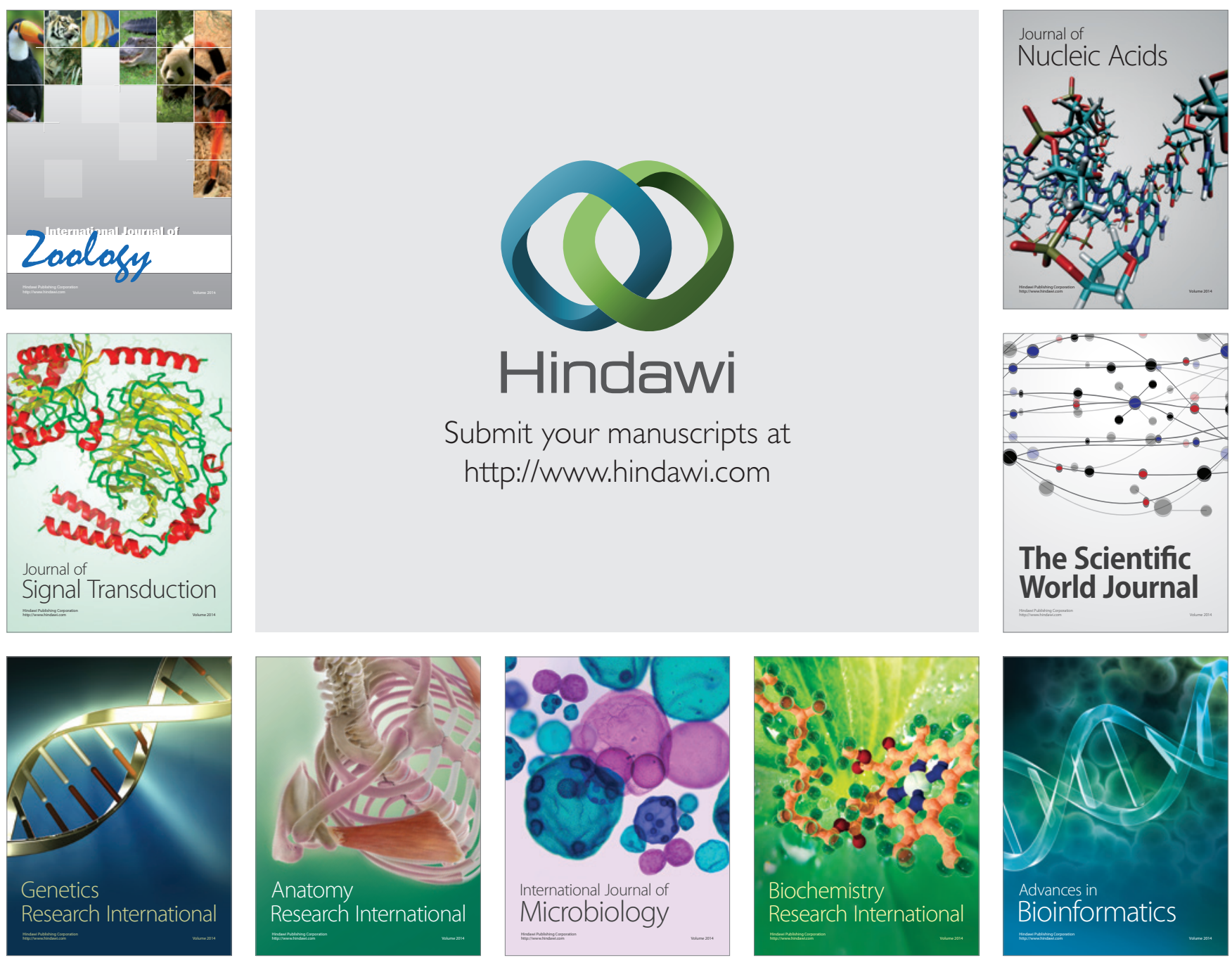

The Scientific World Journal
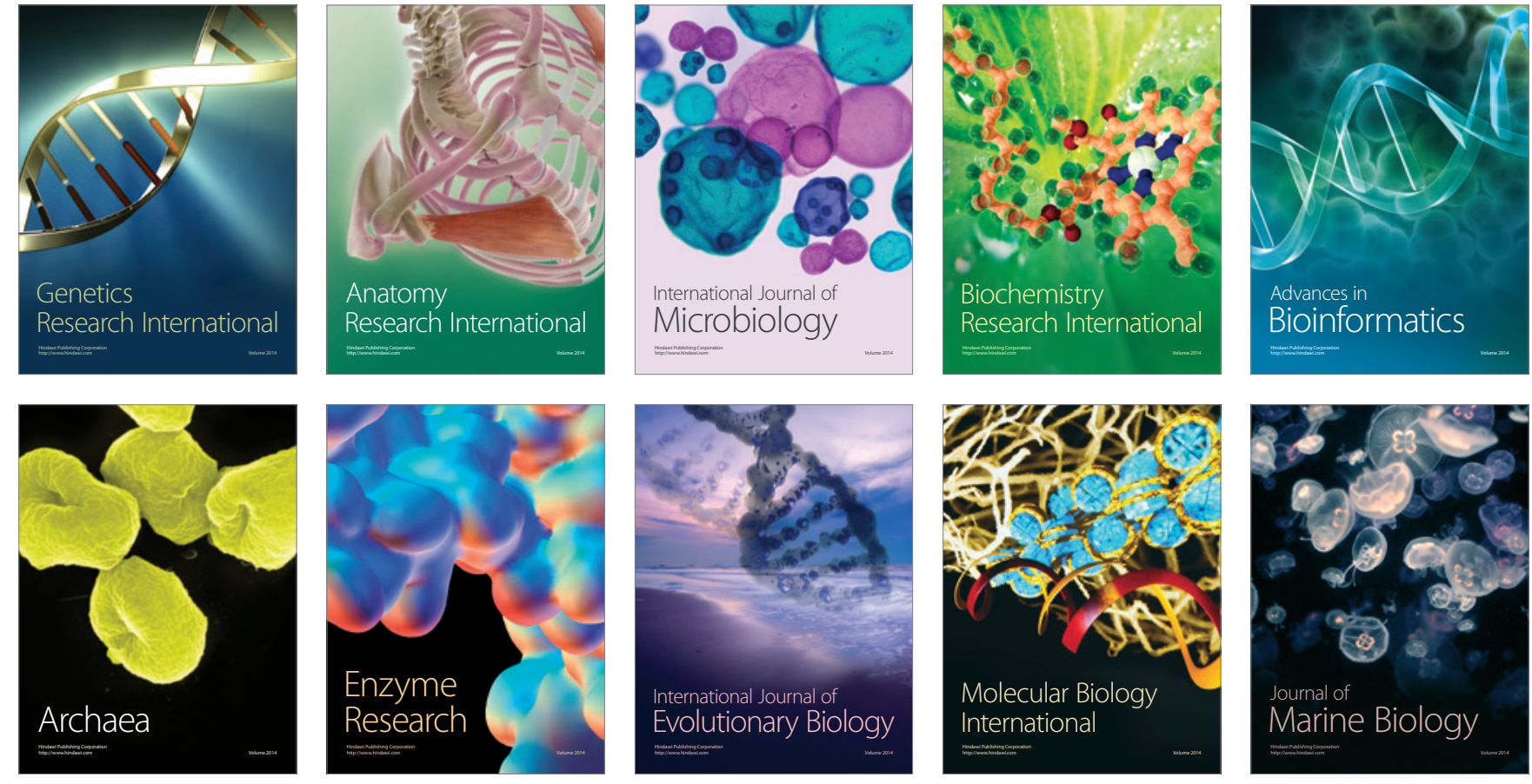